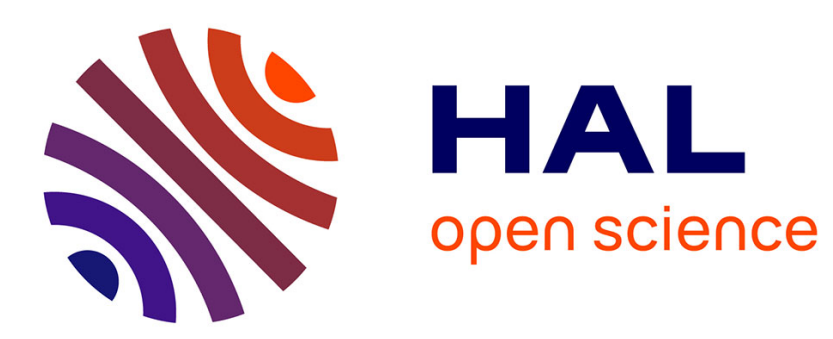

\title{
A new procedure for the determination of structural characteristics of sandwich plates in medium frequencies
}

Christophe Droz, Olivier Bareille, Mohamed N Ichchou

\section{To cite this version:}

Christophe Droz, Olivier Bareille, Mohamed N Ichchou. A new procedure for the determination of structural characteristics of sandwich plates in medium frequencies. Composites Part B: Engineering, 2017, 112, pp.103-111. 10.1016/j.compositesb.2016.12.023 . hal-03373007

\section{HAL Id: hal-03373007 https://hal.science/hal-03373007}

Submitted on 11 Oct 2021

HAL is a multi-disciplinary open access archive for the deposit and dissemination of scientific research documents, whether they are published or not. The documents may come from teaching and research institutions in France or abroad, or from public or private research centers.
L'archive ouverte pluridisciplinaire HAL, est destinée au dépôt et à la diffusion de documents scientifiques de niveau recherche, publiés ou non, émanant des établissements d'enseignement et de recherche français ou étrangers, des laboratoires publics ou privés. 


\title{
A new procedure for the determination of structural characteristics of sandwich plates in medium frequencies
}

\author{
Christophe Droz ${ }^{\mathrm{a}, 1}$, Olivier Bareille ${ }^{\mathrm{a}}$, Mohamed N. Ichchou ${ }^{\mathrm{a}}$ \\ ${ }^{a}$ École Centrale de Lyon, 36 Avenue Guy de Collongue, 69134 Écully Cedex, France
}

\begin{abstract}
This paper presents a new wave-based material identification strategy for sandwich panels, able to estimate their tensile and equivalent shear moduli from single shot measurements. The proposed algorithm is based on the determination of the maximal wave speed in the bending-to-shear transition bandwidth. A definition is proposed for the transition phenomenon resulting from the conversion of flexural waves in the composite waveguide, and the existence of a local energy velocity maxima is demonstrated using the $4^{\text {th }}$-order sandwich theory. Analytical expressions are derived for the equivalent bending and shear parameters in terms of the transition frequency and the maximal group velocity. An practical iterative procedure is also proposed for the experimental identification of the transition bandwidth, while a Hilbert Transform magnitude is employed to measure time of arrivals of the flexural wave pulses. The robustness of the method is highlighted using a numerical case study and an experimental validation is finally conducted on a sandwich panel with composite faces. Results are compared with static measurements and a wave-based technique (Inhomogeneous Wave Correlation) and show significant accuracy improvements while ensuring reduced post-processing times.
\end{abstract}

Keywords: Honeycomb, sandwich panel, inverse identification, group velocity, wave

\section{Introduction}

Composite assemblies have become a crucial component in transportation and aerospace industries due to their exceptional strength-to-weight ratios. Sandwich panels have the most widespread utilization due to their wide range of possible configurations. They are usually composed of a soft and lightweight core made of foam or honeycomb construction, surrounded by thin fibrereinforced skins. However, the design and optimization of these structures in vibroacoustic environments remain challenging, especially due to the difficulty to perform an accurate modelling of the composite assemblies in the medium frequency range [1], too high for deterministic finite element models to apply while the main assumptions of energy distribution underlying the Statistical Energy Analysis (SEA) are not met [2]. Yet, although the design of structurally advanced

Email address: christophe.droz@gmail .com (Christophe Droz )

${ }^{1}$ Current address: KU Leuven, Department of Mechanical Engineering, Division PMA, Celestijnenlaan 300 - box 2420, Leuven, Belgium 
composites does not usually requires models of the local scale's components (such as the aramid fibers of a honeycomb construction, or the resin of its skins), accurate predictions of the overall dynamical behaviour at the global scale are fundamental.

Although a large number of mechanical characteristics of sandwich's components can be found in the literature, the increasing research effort on the development of composite skins and structurally advanced core's constructions yields a challenge in terms of prediction of the equivalent core's and skin's material parameters [3]. Besides, the same nominal sandwich panel (same core size, materials and stacking sequence) can exhibit different mechanical characteristics, depending on the manufacturing process or epoxy resin density, which can reach more than $30 \%$ of the total mass. Additionally, the use of material tables defined using static experiments for the modelling of sandwich panels in the vibroacoustic range can become an issue. Indeed, although frequencyindependence of the mechanical parameters is a fair assumption in a given bandwidth, static and frequency identifications often show great discrepancies.

Various methods were devoted to the determination of effective core properties of sandwich panels $[4,5]$, Karakoç and Freund [6] proposed an experimental procedure to determine the compliance matrix of Nomex structures. For dynamical identification, modal methods are often employed, Matter et al. [7] proposed an identification technique for evaluating the elastic and damping properties of sandwich laminates with soft cores. Other methods based on free modes and comparisons with analytical and FE methods were also developed $[8,9,10]$ to determine elastic properties of sandwich structures. Recently, wave-based methods were developed for the determination of these equivalent properties. They advantageously avoid the uncertainties produced by the boundaries on the modal responses and enable effective characterization at higher frequencies, where modal overlap becomes too important to use modal data (see [11] for an example of high-frequency characterization of polypropylene composites).

The propagation of guided waves in sandwich structures has indeed been the subject of intense research in the recent years. Many studies are concerned with exploiting wave dispersion characteristics (also called dispersion curves) to understand wave's physics in heterogeneous structures and design composite waveguides with advanced capabilities [12,13]. One can cite, among other: the design of auxetic pyramidal cores [14], the vibroacoustic optimization of sandwich's geometries [15, 16], or the inclusion of internal resonators to improve damping properties [17] and a very large number of applications $[18,19]$ can also be found for defect detection in sandwich structures.

For these new types of composites, studies were conducted on experimental wave-based methods for the identification of effective elastic properties of sandwich constructions: an inverse wave approach for estimating structural wave parameters was proposed by McDaniels and Shepard [20]. The Inhomogeneous Wave Correlation (IWC) method was proposed in [21] for the identification of the dispersion curves in two dimensions, also called k-space. Least mean square algorithm is then applied to sandwich plate theory to obtain the homogenized panel properties. Despite a rather good accuracy, these methods require to measure and post-process the entire displacement field to extract the wavenumber space, leading to expensive and time-consuming characterizations. One of the consequences is a possible inaccuracy when using standard sandwich material tables provided in the literature.

This paper addresses the genuine need of wave-based mid-frequency identification methods for sandwich panels, involving simple experimental set-ups. A strategy is investigated for the 
elastic parameter identification of sandwich panels using Time-of-Flight (ToF) measurements [22, 23 ], since measuring the energy velocity in plates is a mature technique extensively used in the literature (interested reader may refer to [24, 25, 26] or Sikdar et al. [27] for a recent application to honeycomb composites). The proposed method focuses on a phenomenon called transition, which is a specific type of wave conversion in waveguides [28, 29], occurring in sandwich structures subjected to flexural vibrations.

In section 2, the practical consequences of the transition on flexural waves' velocities are studied and an definition based on $4^{\text {th }}$-order sandwich theory is proposed for the transition frequency. The existence of a local velocity maximum around this frequency is exploited to dissociate the shear and bending contributions to the wave motion in the composite. An identification procedure based on transient wave pulses velocities is proposed in section 3 and applied to a sandwich waveguide. The numerical results are compared with analytical results and the accuracy of the identification method is discussed. In section 4, an experimental case study is proposed. The mechanical characteristics of a sandwich panel involving a Nomex honeycomb core surrounded with carbon-reinforced skins are estimated using the proposed method and compared with the IWC results and manufacturer's data.

\section{Transition frequency in symmetric sandwich}

\subsection{Theoretical background}

The governing equations for the anti-symmetric motion of sandwich waveguides are described hereby $[30,31]$. Consider a sandwich plate whose normal deflection is assumed connected to the core and shear rotations $\beta$ and $\gamma$. The total deflection $w$ in the direction $x$ produced by $\beta$ and $\gamma$ is:

$$
\frac{\partial w}{\partial x}=\beta+\gamma
$$

Considering a linear Lagrangian $H=T-U$, where $U$ and $T$ stand for the potential and kinetic energies, Hamilton's principle yields the following variational expression for a conservative system :

$$
\delta \int H \mathrm{~d} t=0
$$

Combining Eqs.(1) and (2) gives the differential equations for $\beta$ and $w$ :

$$
\begin{array}{r}
2 D_{2}\left(\frac{\partial^{4} w}{\partial x^{4}}-\frac{\partial^{3} \beta}{\partial x^{3}}\right)-G_{c} h_{c}\left(\frac{\partial^{2} w}{\partial x^{2}}-\frac{\partial \beta}{\partial x}\right)+\mu \frac{\partial^{2} w}{\partial t^{2}}=0 \\
2 D_{2}\left(\frac{\partial^{3} w}{\partial x^{3}}-\frac{\partial^{2} \beta}{\partial x^{2}}\right)-D_{1} \frac{\partial^{2} \beta}{\partial x^{2}}+I_{\rho} \frac{\partial^{2} \beta}{\partial t^{2}}-G_{c} h_{c}\left(\frac{\partial w}{\partial x}-\beta\right)=0
\end{array}
$$

where $G_{c}$ is the core's shear modulus, $h_{c}$ and $h_{s}$ are the core's and skin's thickness. The overall flexural stiffness $D_{1}$, the layer's flexural stiffness $D_{2}$ and the mass moment of inertia per unit width 
$I_{p}$ and mass per unit area $\mu$ are written:

$$
\begin{aligned}
D_{1}=E_{c} \frac{h_{c}^{3}}{12}+E_{s} h_{s}\left(\frac{h_{c}^{2}}{2}+h_{c} h_{s}+\frac{2 h_{s}^{2}}{3}\right), & D_{2}=E_{s} \frac{h_{s}^{3}}{12}, \\
I_{p}=\rho_{c} \frac{h_{c}^{3}}{12}+\rho_{s} h_{s}\left(\frac{h_{c}^{2}}{2}+h_{c} h_{s}+\frac{2 h_{s}^{2}}{3}\right), & \mu=2 h_{s} \rho_{s}+h_{c} \rho_{c} .
\end{aligned}
$$

where $E_{s}$ and $\rho_{s}$ are the skin's Young modulus and density, and $\rho_{c}$ is the equivalent core's density.

Eliminating the angular displacement $\beta$ in Eqs.(5) and 6) and assuming plane waves propagate along the $\mathrm{x}$-direction : $w(x, t)=w_{0} \mathrm{e}^{-\mathrm{j}(\omega t-k x)}$, with $k$ the wavenumber and $\omega$ the circular frequency yields:

$$
2 D_{1} D_{2} k^{6}-2 D_{2} I_{\rho} k^{4} \omega^{2}-\left(\mu+2 D_{2} \mu+I_{\rho} G_{c} h_{c}\right) k^{2} \omega^{2}+G_{c} h_{c} D_{1}\left(k^{2}-\mu \omega^{2}\right)+I_{\rho} \mu \omega^{4}=0
$$

Two main assumptions are introduced: the rotation inertia is negligible compared with the shear effects $I_{\rho} \omega^{2} \ll G h_{c}$, and the bending stiffness per unit width of the skins are small in comparison with the one of the plate: $D_{2} \ll D_{1}$. Therefore the $4^{\text {th }}$-order dispersion relation in the sandwich becomes:

$$
S k^{4}-\mu \omega^{2} k^{2}-\mu \omega^{2} S / D=0
$$

where $S=h_{c} G_{c}\left(1+\frac{h_{s}}{h_{c}}\right)^{2}$ and $D \simeq E_{s} h_{s}\left(\frac{h_{c}^{2}}{2}+h_{c} h_{s}+\frac{2 h_{s}^{2}}{3}\right)$ are the transverse shear rigidity and bending stiffness of the plate. Note that Eqs.(1-8) are derived for propagation in $x$-direction. In the case of a honeycomb sandwich with composite face sheets, the equivalent bending and shear coefficients depend on the considered direction $k_{\theta}=f\left(\mu, S_{\theta}, D_{\theta}\right)$.

\subsection{Asymptotic solutions for shear and bending modulus identification}

The solution of Eq.(8) gives the flexural wavenumber:

$$
k^{2}=\frac{\mu}{2 S}\left(\omega^{2}+\omega \sqrt{\omega^{2}+\frac{4 S^{2}}{\mu D}}\right)
$$

When $D, S$ and $m$ are fixed, two asymptotic behaviours can be distinguished: at low frequency, the sandwich plate is ruled by the skins' Young modulus and has classical plate behaviour. The 'bending' wavenumber and group velocity are written:

$$
k_{\text {bending }}=k(\omega \rightarrow 0)=\sqrt[4]{\frac{\mu}{D}} \sqrt{\omega}, \quad ; \quad c_{g}(\omega \rightarrow 0)=2 \sqrt[4]{\frac{D}{\mu}} \sqrt{\omega}
$$

while in higher frequencies the sandwich's motion is mainly governed by the shear coefficient $S$. The asymptotic wavenumber and group velocity become:

$$
k_{\text {shear }}=k(\omega \rightarrow \infty)=\sqrt{\frac{\mu}{S}} \omega, \quad ; \quad c_{g}(\omega \rightarrow \infty)=\sqrt{\frac{S}{\mu}} .
$$


Between these two frequency domains, both the shear and bending modulus contribute to the flexural motion. In sandwich structures, the transition frequency refers to the passage from a flexural wave governed by the skins' stiffness to one governed by the core's transverse shear. The asymptotic expressions given in Eqs.(10) and (11) were used by Ghinet and Atalla [32] and more recently in $[15,33,34]$ to give interpretations of the transition in sandwich panels. In [33], the transition frequency $\omega_{T}$ was defined by the geometric intersection between the asymptotic wavenumbers, $k_{\text {bending }}\left(\omega_{T}\right)=k_{\text {shear }}\left(\omega_{T}\right)$, leading to a transition frequency $\omega_{T}=\frac{S}{\sqrt{\mu D}}$. It should be noted that a similar definition based on the asymptotic modal densities [32] (or group velocities) yields: $\omega_{T}=\frac{1}{2} \frac{S}{\sqrt{\mu D}}$.

\subsection{Transition and maximum wave velocity}

The definitions given above for the transition can be used for example to define the bandwidth on which regression models based on Eqs.(10) and (11) can be applied. It avoids the use of nonlinear regression models based on Eq.(8) but requires a priori knowledge of the transition to ensure the accuracy. Although the effective bending modulus can be approximated from the dispersion curves below the transition, this is not the case for the shear modulus. Indeed, the shear velocity cannot be measured in sandwich plates due to the presence of the second transition, shown in figure 1a. This transition is associated with a localization of the flexural wave in the skins and occurs once the hypothesis $D_{2} \ll D_{1}$ is no longer applicable. Consequently the asymptotic velocities cannot be measured and used to determine a core's shear coefficient.

However, it can be seen that the group velocity reaches a local maximum at $1 \mathrm{kHz}$. Since the group velocity describes the average local velocity of energy transport in the wave diffusion process, the physical meaning of this value can be seen as the maximum energy flux for a normalized energy density [35].

Since the shear velocity is not reachable, this maximum of energy velocity is the only point of the dispersion curves which is independent of the bending stiffness within the transition bandwidth (see figure 1b.), hence can be considered a pure core's shear effect. In the following, the transition is defined by the frequency at which the wave velocity reaches this local maximum. With this definition, the group velocity can be written from Eq.(9):

$$
c_{g}=\frac{\partial \omega}{\partial k}=\frac{2 \Omega \sqrt{\frac{\mu}{2 S}\left(\omega \Omega+\omega^{2}\right)}}{\frac{\mu}{2 S}\left(2 \omega \Omega+\Omega^{2}+\omega^{2}\right)},
$$

where $\Omega=\sqrt{\omega^{2}+\omega_{0}^{2}}$ and $\omega_{0}=\frac{2 S}{\sqrt{\mu D}}$. The transition frequency defined above requires the frequency derivative of the velocity, written:

$$
\frac{\partial c_{g}}{\partial \omega}=\frac{\omega^{2} \Omega-\Omega^{3}+2 \omega_{0}^{2} \omega}{\left(\omega^{2} \Omega+\Omega^{3}+2 \omega_{0} \omega^{2}+2 \omega^{3}\right) \sqrt{\frac{\mu}{2 S}\left(\omega \Omega+\omega^{2}\right)}}
$$



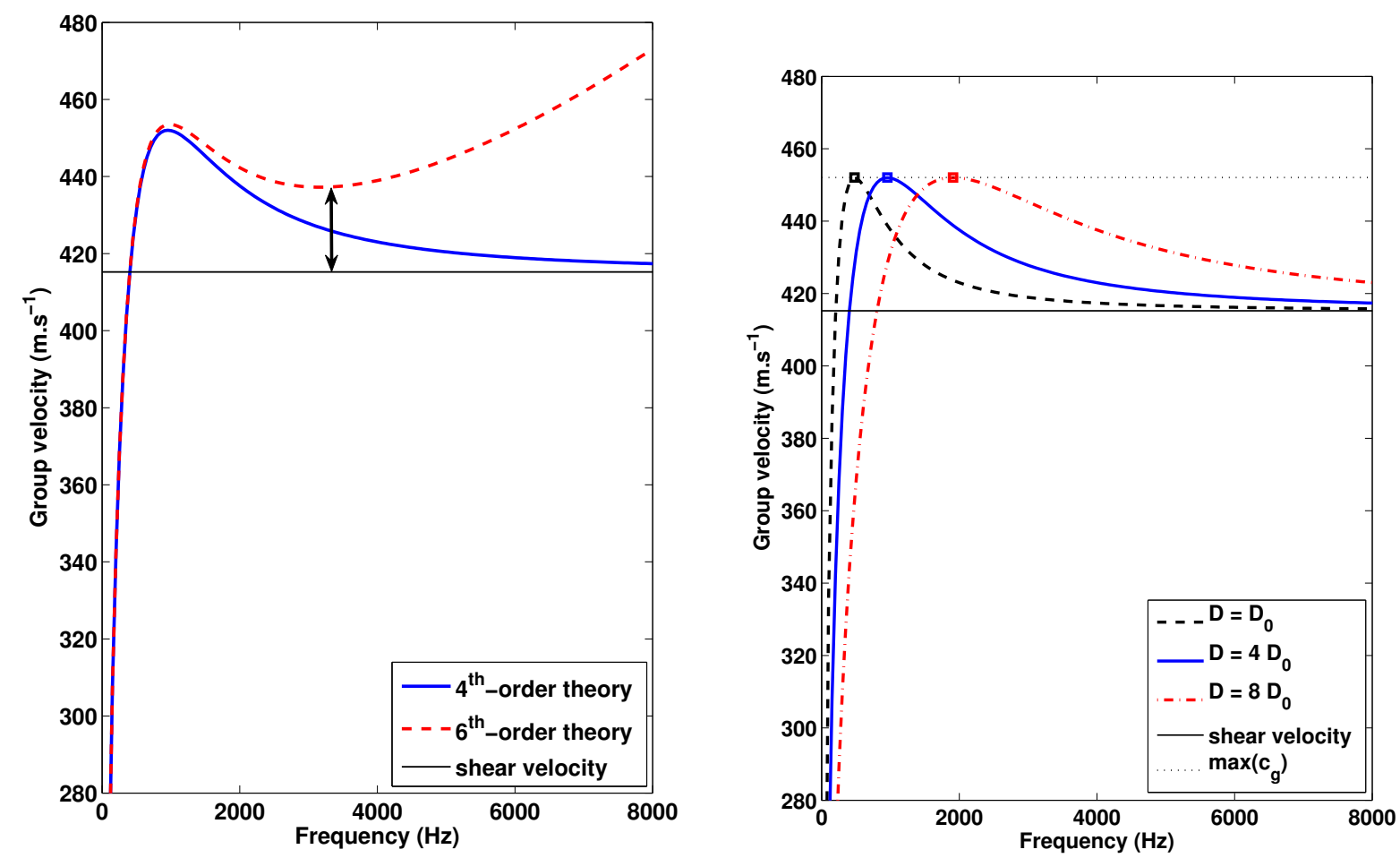

Figure 1: (a) Comparison of the group velocities obtained through $4^{\text {th }}$ (—) and $6^{\text {th }}$-order theory $(---)$. Sandwich parameters: $D / \mu=1100 \mathrm{~m}^{4} \cdot \mathrm{s}^{-4}, S / \mu=172420 \mathrm{~m}^{2} \cdot \mathrm{s}^{-2}, h_{c}=10 \mathrm{~mm}, h_{s}=0.6 \mathrm{~mm}$.

(b) Influence of the bending modulus $\left(D_{0}=2653 \mathrm{Nm}\right)$ on the frequency and value of the maximal group velocity. Shear velocity: $c_{\text {shear }}=415.2 \mathrm{~m} . \mathrm{s}^{-1}$, maximum velocity $c_{\max }=452 \mathrm{~m} \cdot \mathrm{s}^{-1}$.

Solving $\frac{\partial c_{g}}{\partial \omega}=0$ gives the solution for the transition frequency:

$$
\omega_{T}=\frac{2 S}{\sqrt{3 \mu D}} .
$$

Therefore the maximum group velocity is written:

$$
c_{g}\left(\omega_{T}\right)=\frac{4}{3} \sqrt{\frac{2 S}{3 \mu}} .
$$

Noteworthy, the velocity at the transition is independent of the bending stiffness $D$, as observed in figure 1b. The shear coefficient $S$ can therefore be determined without the use of regression models requiring the entire dispersion curves in the considered bandwidth. Besides, the asymptotic and transition velocities are related by the coefficient $\frac{c_{g}\left(\omega_{T}\right)}{c_{s}}=\left(\frac{4}{3} \sqrt{\frac{2}{3}}\right)$ which is independent of the sandwich material parameters. This standard exceedance of approximately $8.87 \%$ of the shear 
velocity can lead to a significant underestimation of the time-of-flight in the transition bandwidth. As the maximal energy velocity of a wave pulse can be easily determined in a given frequency range, the shear coefficient can be derived using the following expression:

$$
S=\frac{27 \mu}{32} \max \left(c_{g}\right)^{2}
$$

The bending modulus can then be determined using Eqs.(13) and (15):

$$
D=\frac{4 S^{2}}{3 \mu \omega_{T}^{2}}
$$

Both $S$ and $D$ can therefore be determined in the direction of propagation using the maximal velocity and its frequency in a sandwich panel. In the following, a numerical validation is proposed for a sandwich waveguide whose material characteristics are known.

\section{Numerical validation}

In this section, the group velocity and transition frequency are determined in a sandwich structure to identify its equivalent mechanical characteristics. Consider the waveguide defined in table 1. Vertical motion is applied at $x=X_{0}$ to produce a flexural motion while the displacements are acquired at $x=X_{1}$ where $X_{1}-X_{0}=3 \mathrm{~m}$. The excitation signal is a tone burst of amplitude $U_{0}=10^{-6} \mathrm{~m}$ involving $n_{0}$ cycles at the frequency $f_{0}$ with Hanning window defined by:

$$
u(t)= \begin{cases}U_{0} \sin \left(\frac{\pi f_{0} t}{n_{0}}\right) \sin \left(2 \pi f_{0} t\right), & t \leq \frac{n_{0}}{f_{0}} \\ 0, & t>\frac{n_{0}}{f_{0}}\end{cases}
$$

\begin{tabular}{|l|c|c|c|c|}
\hline Material & Thickness & Density $\left(\mathrm{kg} \cdot \mathrm{m}^{-1}\right)$ & Young modulus (GPa) & Shear modulus (MPa) \\
\hline Skin & $0.3 \mathrm{~mm}$ & 1451 & $E_{s}=81$ & $G_{s}=2780$ \\
\hline Core & $25 \mathrm{~mm}$ & 53 & $E_{c}=5.23$ & $G_{c}=50$ \\
\hline
\end{tabular}

Table 1: Dimensions and material characteristics of the sandwich beam. Note that the core is described as an homogeneous medium. The beam's width is $l_{y}=10 \mathrm{~mm}$ and the damping is not considered in this model.

Note that the value of $n_{0}$ has a significant influence on the bandwidth of the excitation spectrum, hence on the possible spatial dispersion of the wave pulses. An illustration of the acquisition procedure is given in figure 2 . The propagation of wave pulses with different frequencies and durations is computed using FEA on a distance of $3 \mathrm{~m}$. The Hilbert Transform $H[x(t)]$ of a time signal $x(t)$ is commonly used to calculate the main attributes of $x(t)$. It is defined by:

$$
H[x(t)]=\frac{1}{\pi} \int_{-\infty}^{+\infty} \frac{x(t)}{t-\tau} d \tau .
$$




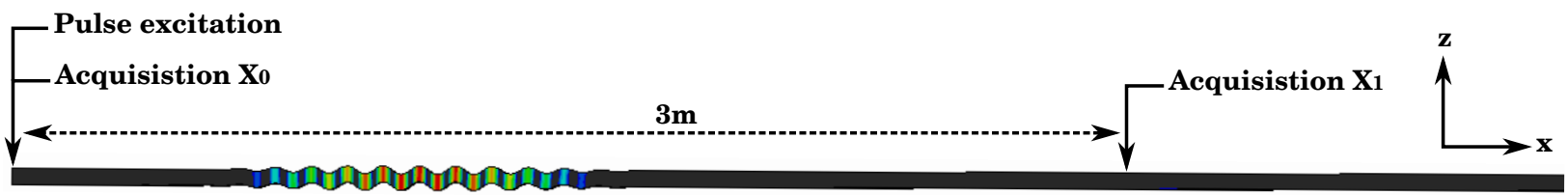

Figure 2: Illustration of the numerical procedure for measuring the group velocities in the beam. Simulations are conducted using the FEA package Abaqus CAE V6.13 with Abaqus/Explicit solver. Linear C3D8 elements are used and a minimum mesh density of 15 elements per wavelength is applied, leading to a 120000 elements model at $13.2 \mathrm{kHz}$. Note that the choice of a beam is motivated by the elevated computational times associated with 2D transient simulations.
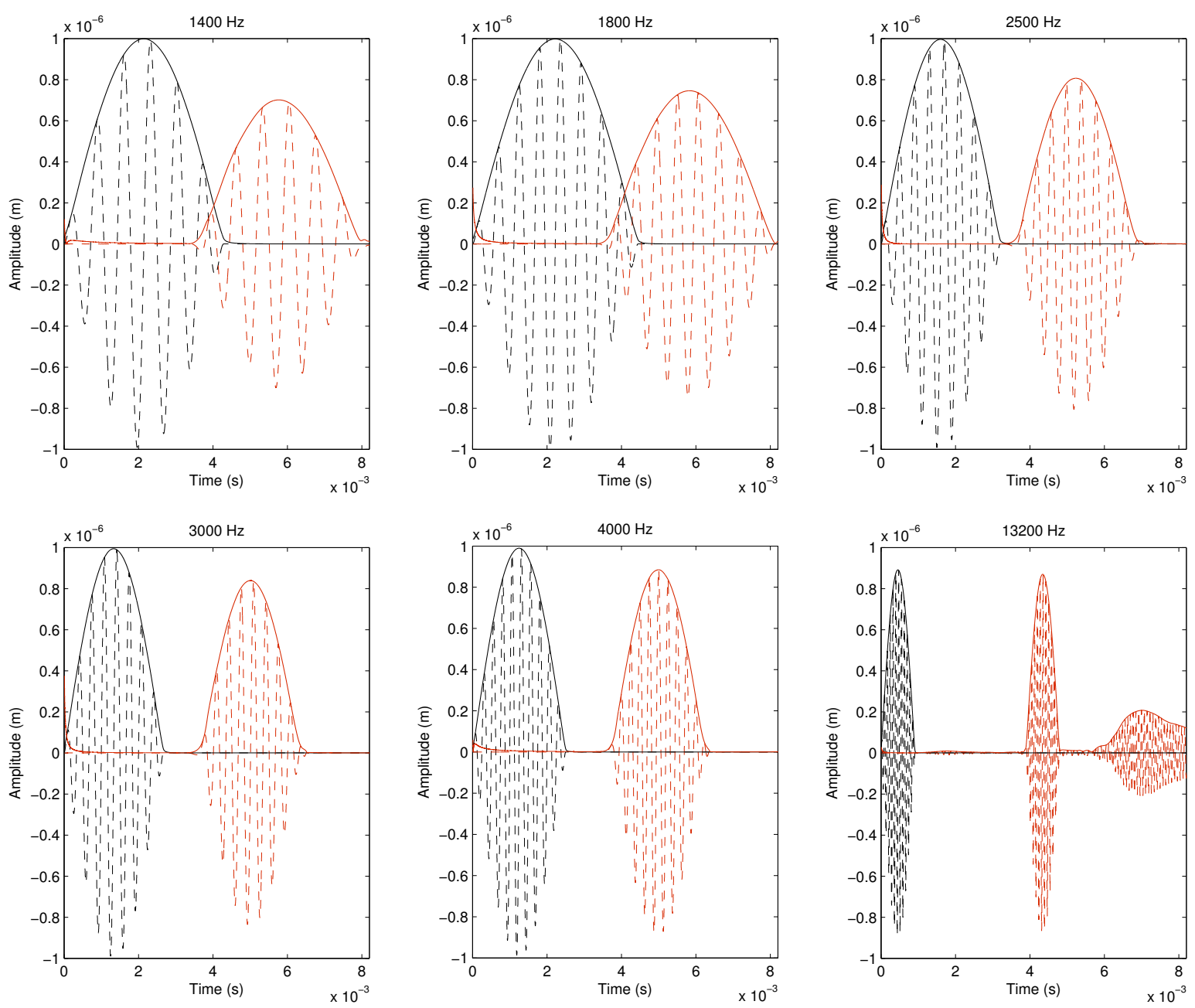

Figure 3: Time acquisition at $x=0(--)$ and $x=3 \mathrm{~m} \mathrm{(--)}$ in the sandwich waveguide. The number of cycles is: 6 at $1400 \mathrm{~Hz}, 8$ between $1.8 \mathrm{kHz}$ and $3 \mathrm{kHz}, 10$ at $4 \mathrm{kHz}$ and 12 at $13.2 \mathrm{kHz}$. The time of flight is measured at the maximal amplitude of Hilbert transform (solid lines) signal.

The signal envelope is determined at emission, $X_{0}$ and arrival, $X_{1}$ using Hilbert transform while the time delay is defined by the time difference between the maximal amplitudes of the envelopes. 
Results are shown in figure 3 for six wave pulses with different frequencies. At $1.4 \mathrm{kHz}$ the pulse exhibits higher dispersion due to the reduced number of cycles, while negligible dispersion occurs above $4 \mathrm{kHz}$. A converted wave is observed at $13.2 \mathrm{kHz}$, due to the application of a vertical motion at the free edge, which is not associated with the shear wave motion. The accuracy obtained on the wave's speed clearly increases with frequency, although the absence of reflected and converted waves at low frequencies allows a reliable determination of the envelope's maximum. Exact values for the equivalent bending and shear coefficients can be obtained from Eqs.(5) and (6): $D=7,778 \mathrm{Nm}, S=1.2805 \times 10^{6} \mathrm{~N} / \mathrm{m}$ and the mass per unit area is $\mu=2.196 \mathrm{~kg} \cdot \mathrm{m}^{-2}$. The group velocities obtained through $4^{\text {th }}$-order theory are compared with the transient simulations in figure 4.

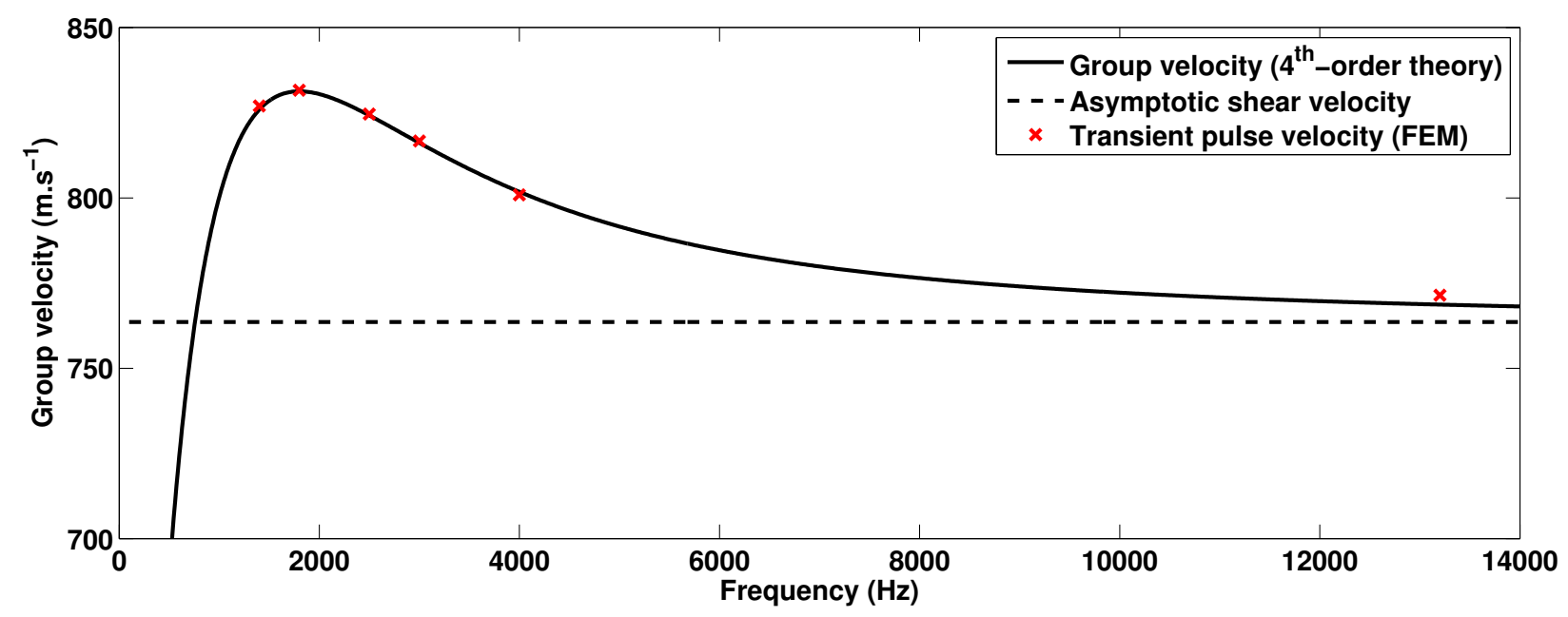

Figure 4: Comparison between theoretical dispersion curves and the velocities obtained by ToF with transient pulse simulations at $1.4 \mathrm{kHz}, 1.8 \mathrm{kHz}, 2.5 \mathrm{kHz}, 3 \mathrm{kHz}, 4 \mathrm{kHz}$ and $13200 \mathrm{kHz}$. The half-amplitude bandwidth of the excitation spectrum varies from $396 \mathrm{~Hz}$ for the first pulse $(1.4 \mathrm{kHz})$ to $1870 \mathrm{~Hz}$ for the last pulse $(13.2 \mathrm{kHz})$.

Noteworthy, the velocities obtained thought transient response are in very good agreement with the analytical results. The accuracy is good, considering the frequency spectrum bandwidth of the excitation signals (only 6 cycles at $1.4 \mathrm{kHz}$ ). A $1 \%$ error is observed at $13.2 \mathrm{kHz}$, which is consistent with the appearance of a second transition frequency (around $14 \mathrm{kHz}$ ) resulting in a dissociation from the asymptotic shear velocity. The analytical shear velocity is $c_{s}=763.6 \mathrm{~m} . \mathrm{s}^{-1}$. Using this approximated velocity and Eq.(11) for the determination of $S$ yields a $2.1 \%$ overestimation of the shear coefficient.

The transition occurs at $1.8 \mathrm{kHz}$ and the maximum group velocity is $c_{\max }=831.6 \mathrm{~m} . \mathrm{s}^{-1}$. Using the relations derived in the previous section yields $D=7,792.4 \mathrm{Nm}$ and $S=1.2811 \times 10^{6} \mathrm{~N} / \mathrm{m}$ which reduces the error to $0.05 \%$. The mechanical characteristics of the panel obtained through transition analysis are therefore :

$$
E_{\text {trans }}=81.16 \mathrm{GPa} \text { and } G_{\text {trans }}=50.04 \mathrm{MPa}
$$

which is a very close from the the exact values for $E_{s}$ and $G_{c}$. 
Based on these simulations, the maximal velocity can be used more effectively than the shear velocity to determine equivalent material properties of the considered sandwich panel. It can be noted that the second transition occurs in high frequencies in the considered example: a reduced core's thickness and thicker skins would result in an even more advantageous use of the transition compared with the shear velocity. It should also be mentioned that the results of the simulations presented in figure 4 could be obtained from a fewer number of pulse acquisitions involving broadband spectrum excitations, combined with Fourier Transform to determine the frequency components. Although this approach can produce faster results in simulations, a better accuracy is expected (see section 4) from tunable quasi-monochromatic wave pulses for the identification of the transition frequency.

\section{Experimental case study}

\subsection{Experimental set-up and mechanical characteristics}

The experiment is carried out on a rectangular sandwich panel measuring $60 \mathrm{~cm} \times 288 \mathrm{~cm}$, placed in a vertical position. The constitutive materials are a $10 \mathrm{~mm}$-thick Nomex honeycomb core involving a $3.2 \mathrm{~mm}$ cell size, while propagation is considered in the $\mathrm{W}$-direction. The core is surrounded by $0.6 \mathrm{~mm}$-thick Hexforce skins with multi-axial carbon-reinforced fibres mixed with SR1700 epoxy resin. The density of the skins is $\rho_{s}=1451 \mathrm{~kg} \cdot \mathrm{m}^{-3}$ and the core's density is measured using the overall panel's weight $\rho_{c}=99 \mathrm{~kg} \cdot \mathrm{m}^{-3}$ (manufacturer: $96 \mathrm{~kg} \cdot \mathrm{m}^{-3}$ ). A picture of the components is shown in figure 5 and a description of the experimental set-up is shown in figure 6.

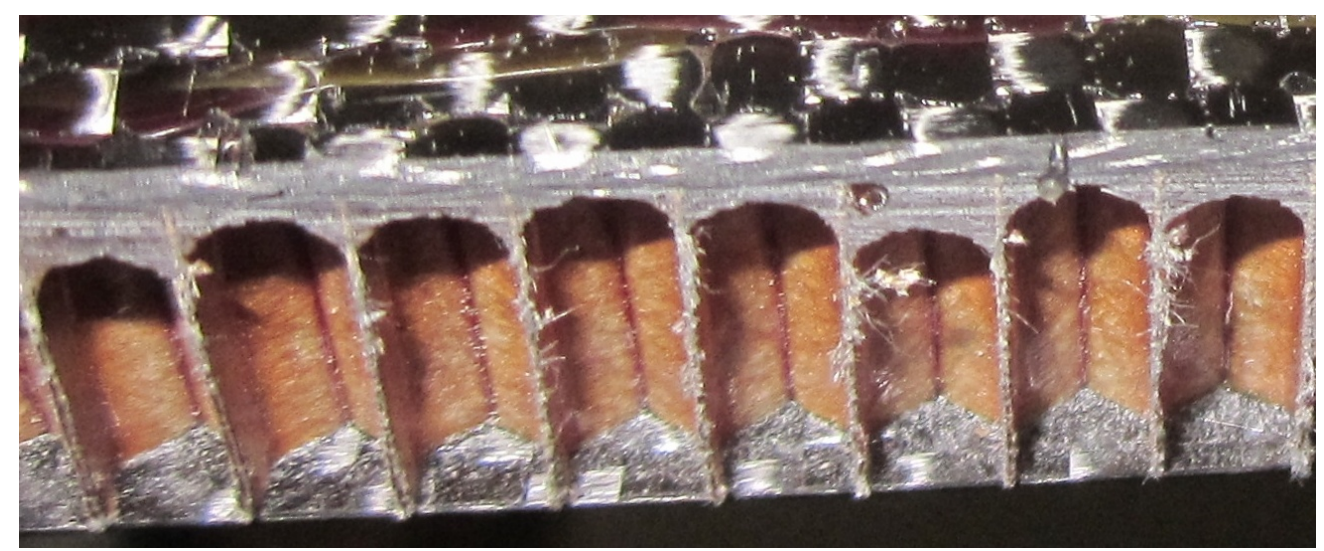

Figure 5: Picture of a section of the sandwich panel, the width is the $W$-direction.

The shaker is placed at the edge of the plate while a laser vibrometer is used to measure normal displacements at the surface. The IWC method [21] is employed to estimate the equivalent shear and bending modulus in the plate. The shaker is used to produce the harmonic excitation while the displacement field is measured using 66 points (referred as IWC measurements in figure 6), distributed along $50 \mathrm{~cm}$, providing a spatial resolution of $7.5 \mathrm{~mm}$ and the wavenumbers between 0 and $4000 \mathrm{~Hz}$. 


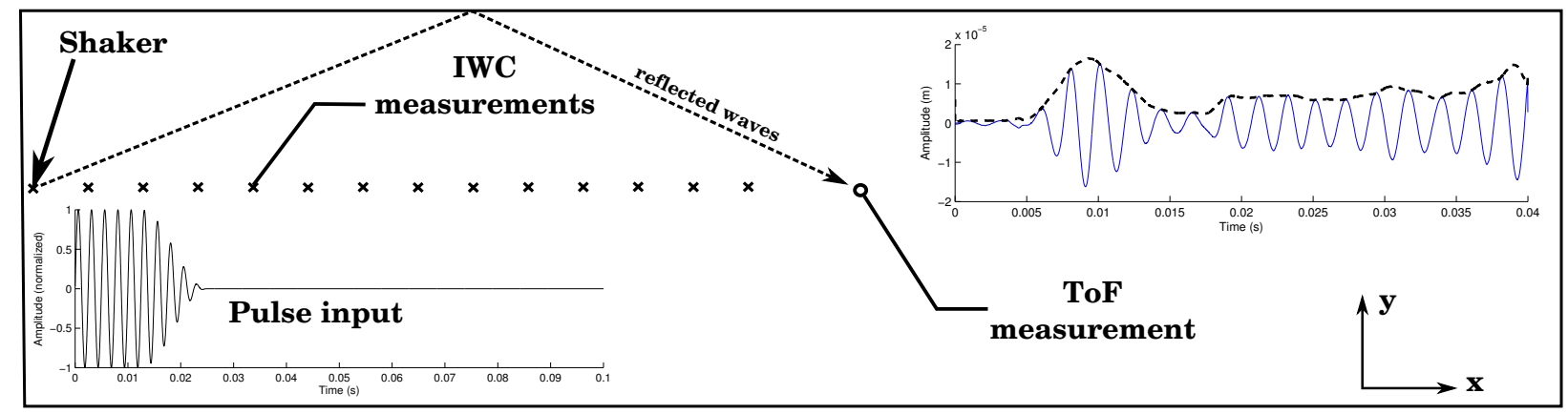

Figure 6: Experimental set-up for the IWC and ToF measurements. The pulse input is generated using the shaker while the time delay is measured at the ToF measurement point. An illustration of the time signal and its Hilbert Transform is shown. Note that due to wave reflection at the edges, it is preferable to use the first phase arrival (FPA) to compute the group velocity, rather than the time delay of the mean wave pulse.

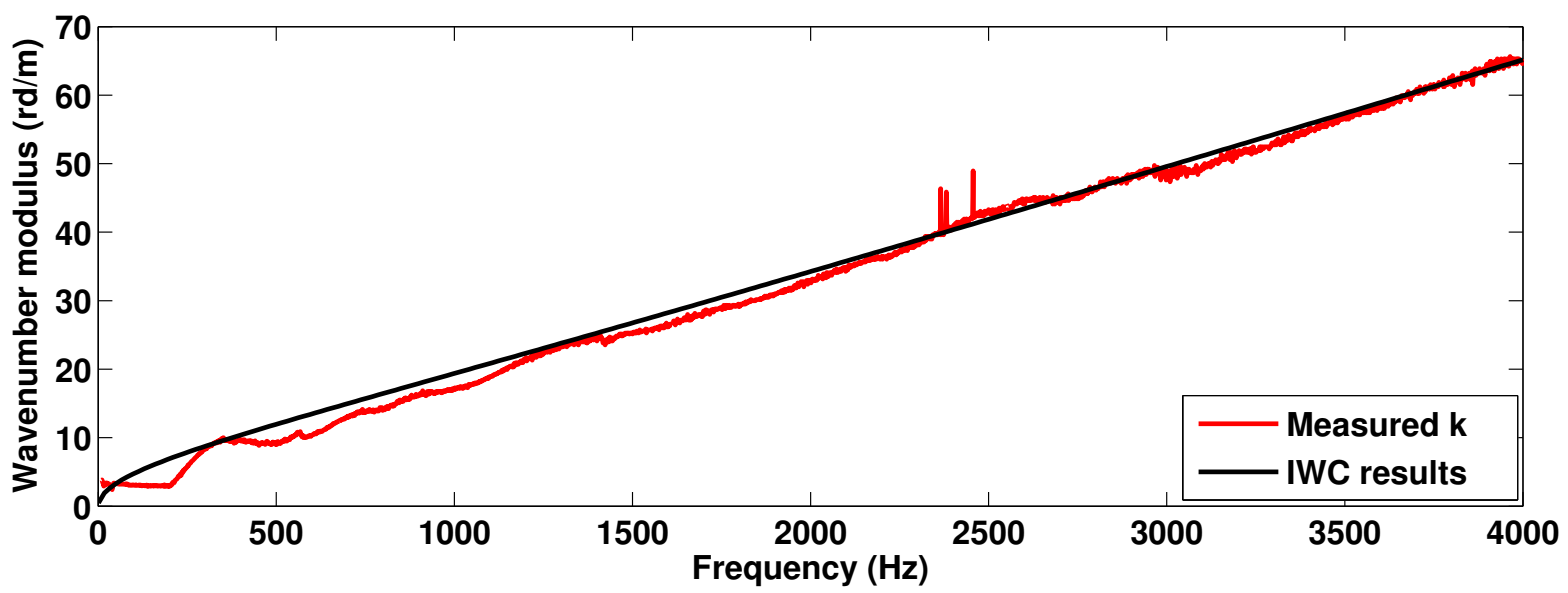

Figure 7: Real wavenumber in the main direction measured in the sandwich plate. A significant error is expected in the low frequencies, due to the limited number of periods for wavelengths determination. The convergence increases in higher frequencies, providing equivalent material characteristics and a good correlation with analytical results.

The wavenumbers measured by the IWC method are shown in figure 7 . The resulting material characteristics are:

$$
E_{\mathrm{iwc}}=62 \mathrm{GPa} \text { and } G_{\mathrm{iwc}}=37.8 \mathrm{MPa}
$$

while the manufacturer provides the following mechanical characteristics:

$$
E_{\text {stat. }}=70 \mathrm{GPa} \text { and } G_{\text {stat. }} \in[30-38] \mathrm{MPa}
$$

which correspond to an $11 \%$ error on $E_{s}$ while $G_{c}$ is located in the upper range of the prediction interval.

\subsection{ToF estimation of the shear and bending coefficients}

The method proposed in section 2 is hereby applied to estimate the Young modulus and shear coefficient from the group velocity at the transition. First, the shaker is used to generate wave 
pulses in the plate at $x=0$ (as shown in figure 6). The duration of the pulse is chosen above 10 cycles to reduce dispersion.

The procedure is conducted as follows: Pulse signals of frequency $\omega$ are generated in the structure and controlled by trigger. The velocity is measured for each pulse at $1 \mathrm{~m}$ from the source using scanning laser vibrometer. The pulse frequency is increased by steps of $20 \mathrm{~Hz}$ between $500 \mathrm{~Hz}$ and $1000 \mathrm{~Hz}$ and $50 \mathrm{~Hz}$ between $1000 \mathrm{~Hz}$ and $1500 \mathrm{~Hz}$ in order to approximate the transition frequency. It is characterized by a decreased of group velocity with frequency. Each pulse is averaged between 30 and 50 times to reduce experimental noise. The proposed procedure is summarized in figure 8. Note that an increased propagation distance $d$ is required for sandwich structures whose transition frequency is low compared with their group velocity.

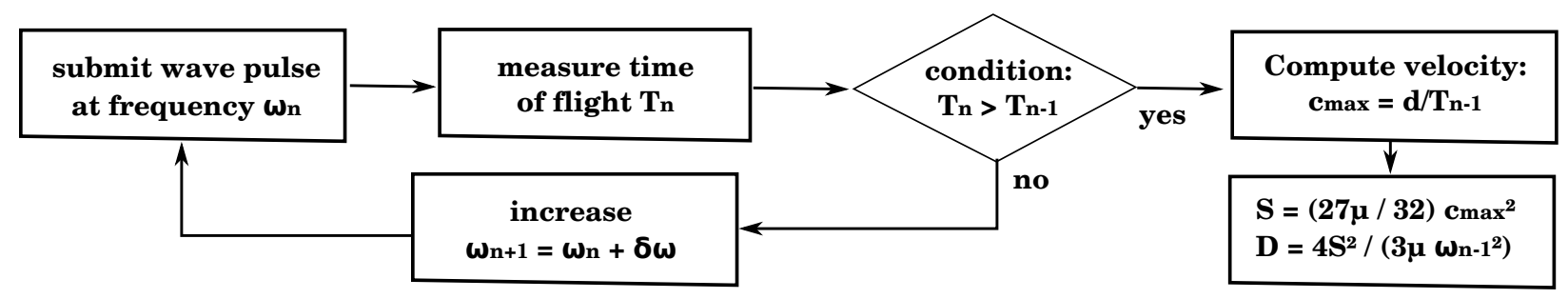

Figure 8: Experimental procedure for the determination of $c_{\max }$ and $\omega_{T}$ summarized as a flow diagram. Note that the comparison between $\mathrm{T}_{n}$ and $\mathrm{T}_{n-1}$ can be achieved on the first arrival of the pulse (FPA), without requiring signal processing and the computation of the group velocity.

Although a first estimation of the transition frequency can be performed without signal postprocessing, it is required for determining the velocity. This step allows an approximation of the transition frequency between $800 \mathrm{~Hz}$ and $950 \mathrm{~Hz}$. A refined sweep is then conducted between $830 \mathrm{~Hz}$ and $900 \mathrm{~Hz}$ to obtain a more accurate value. The time signal is then extracted and postprocessed using Hilbert Transform and compared with the excitation signal to compute the time delay. A comparison between the Hilbert Transform of the time signals for various pulses between $500 \mathrm{~Hz}$ and $1200 \mathrm{~Hz}$ is presented in figure 9.a. It can be seen that the signal amplitude is influenced by the pulse frequency. Above $5 \mathrm{~ms}$, a gap can also be observed between two pulses in the time signal. These effects are produced by the reflections at the edges $(y=0$ and $y=600 \mathrm{~mm})$ and at the free end of the plate $(x=2880 \mathrm{~mm})$. It is therefore clear from figure 9.a that an extensive signal processing work would be required to measure the mean energy velocity while taking into account these reflections.

The main advantage of the proposed method lies in the absence of dispersion of the wave pulse in the forward direction, resulting from the fact that the transition is associated with the maximum group velocity. Indeed, the spectrum contributions of a finite pulse centred on the transition frequency (see for example figure 4) will exhibit velocities below or equal to the maximum group velocity. A practical consequence is a negligible dispersion of the first arriving components of the pulse. It means that the time of flight can be estimated using the difference between the FPA of the input and measured signals.

The Hilbert transform of the FPA is presented in figure 9.b. Noteworthy, a refined frequency sampling for the pulses frequencies and a zoom on the first arrival allows to locate the transition at $880 \mathrm{~Hz}$ with a $10 \mathrm{~Hz}$ accuracy. Further accuracy would be achievable with a better frequency 

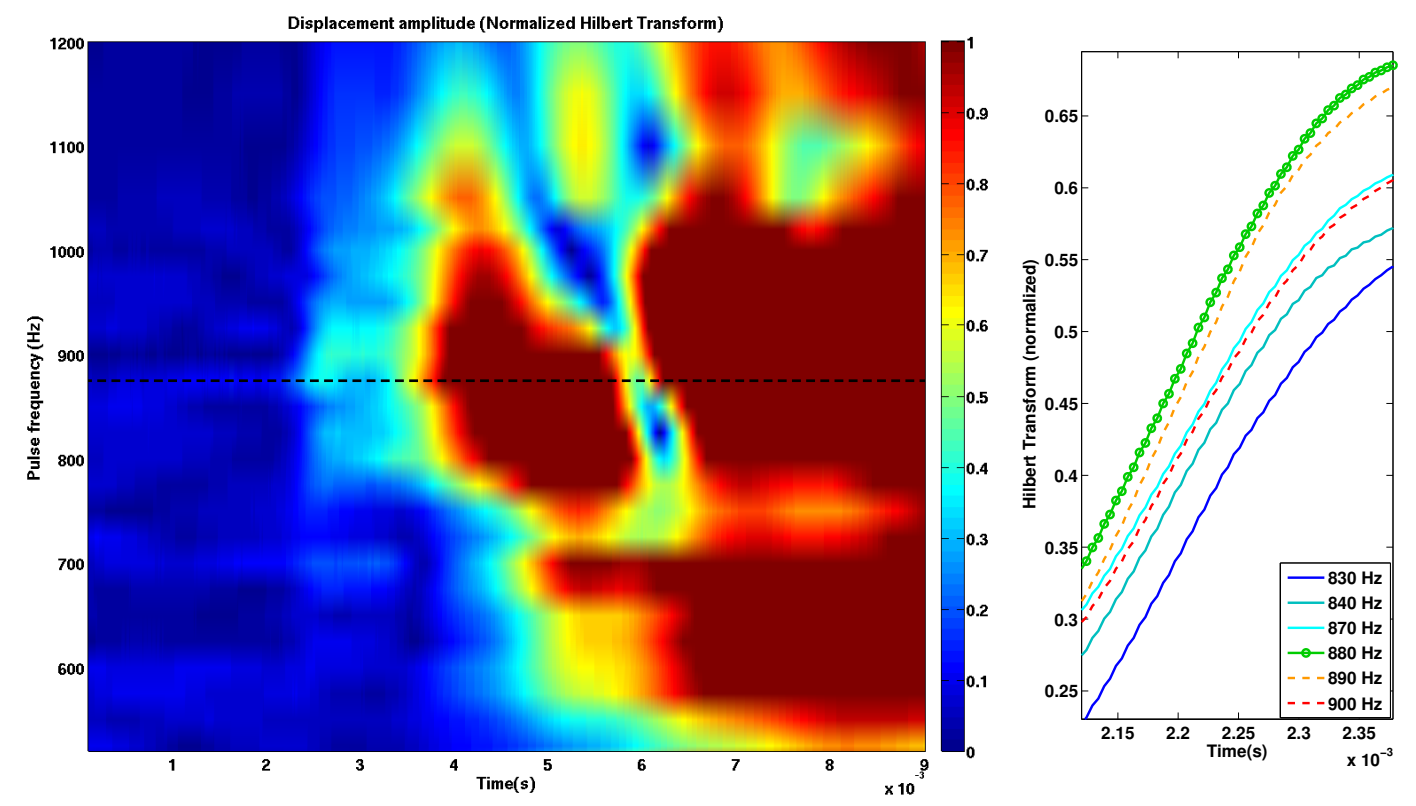

Figure 9: (a): Displacement amplitudes (Hilbert transform) at a distance $x_{0}=1 \mathrm{~m}$ from the excitation source. The amplitude is normalized at $4 \mathrm{~ms}$ to highlight the FPA of the pulse. The first incoming wave pulse is observed at $880 \mathrm{~Hz}$ (- - ) while the time of arrival is $t=2.373 \mathrm{~ms}$. Note that additional filtering procedures can significantly increase the accuracy of the measured velocity (see for example [36, 37]). (b): Comparison of the different measured pulses arrivals between $800 \mathrm{~Hz}$ and $900 \mathrm{~Hz}$. A $10 \mathrm{~Hz}$ resolution is sufficient to obtained a good evaluation of the transition frequency.

resolution, but it will not be needed in the considered case study. Indeed, the second consequence of the dispersion characteristics at the transition is that the maximal velocity will not be affected by inaccuracies on the transition frequency, provided the excitation spectrum bandwidth covers the exact transition. The time of arrival therefore reaches its minimum at $880 \mathrm{~Hz}$ for $t=2.373 \mathrm{~ms}$ after a $1 \mathrm{~m}$ propagation so the maximal velocity is: $c_{\max }=421.4 \mathrm{~m} \cdot \mathrm{s}^{-1}$. Considering the surface density $\mu=2.7402 \mathrm{~kg} . \mathrm{m}^{-2}$ and using Eqs.(15) and (16), the bending and shear coefficients become: $D=2,683 \mathrm{Nm}$ and $S=4.1058 \times 10^{5} \mathrm{~N} / \mathrm{m}$. The equivalent Young modulus of the skins and shear coefficient of the honeycomb core in the main direction are therefore:

$$
E_{\mathrm{ToF}}=69.8 \mathrm{GPa} \text { and } G_{\mathrm{ToF}}=36.5 \mathrm{MPa}
$$

which is a significant improvement compared with the IWC results since $E_{\mathrm{ToF}}$ and $G_{\mathrm{ToF}}$ are both in very good agreement with the manufacturer's values. The authors emphasize that in the case of an orthotropic panel, this procedure only provides the directional values for $E$ and $G$ in the propagating direction.

\section{Concluding remarks}

This paper has explored a new characterization technique based on the wave dispersion characteristics of guided waves in sandwich plates. The method provides the tensile and shear modulus of sandwich structures in the medium-frequency range by taking advantage of the singular 
wave dispersion characteristics of flexural waves within the so-called transition bandwidth. The bending-to-shear transition phenomenon was studied using the WFEM and compared with theory. It was shown that this transition is associated with a local maximum of the group velocity and analytical expressions were derived for the maximal velocity and its frequency. It has also been observed that this maximum wave speed is independent of the shear coefficient.

It is emphasized that the proposed wave-based method has significant advantages: the accuracy of the structural parameters is not altered by the presence of dissipative or uncertain boundaries since the data is obtained locally, through single-shot measurements. It is a considerable advantage compared with a number of stationary or other existing methods, since it can then be applied in situ, without requiring additional sample of the structure. The use of the transition bandwidth results in the absence of wave pulse dispersion in the forward direction of propagation, hence enables the use of an FPA measurement. The consequence is a significant increase of the ToF robustness in low frequency while the post-processing effort becomes almost negligible.

To summarize, the paper resulted in the following findings:

- A definition for the transition phenomenon using the existence of a local energy velocity maxima,

- A FPA strategy for determining this velocity from single shot measurements at low frequencies,

- An in situ procedure for measuring effective mechanical parameters of sandwich plates in the vibroacoustic range.

It can be noted that this strategy however requires the existence of a transition which may not always occur when the skins' thickness is large compared with the core's. The flexural waves in the sandwich are therefore converted into flexural waves in the skins while the core's shear effect is negligible. In general the width does not have any influence on the ToF results. Yet, is some highly directive structures the first incoming phase can be produced by reflections on the edges with higher velocities than in the main direction. A reduction of the distance between the acquisition points is then recommended. Furthermore, note that the proposed method may also apply to other wave types, since it is reminded that the bending-to-shear transition in sandwich panels is a specific type of wave conversion.

[1] Mace, B., Desmet, W., Pluymers, B.. Mid-frequency methods in sound and vibration-part a. Journal of Sound Vibration 2013;332:1895-1896.

[2] Lyon, R.H., DeJong, R.G., Heckl, M.. Theory and application of statistical energy analysis. J Acous Soc Am 1995;98:3021.

[3] Hohe, J., Becker, W.. Effective stress-strain relations for two-dimensional cellular sandwich cores: Homogenization, material models, and properties. Applied Mechanics Reviews 2002;55(1):61-87.

[4] Cunningham, P., White, R.. A new measurement technique for the estimation of core shear strain in closed sandwich structures. Composite structures 2001;51(3):319-334.

[5] Schwingshackl, C., Aglietti, G., Cunningham, P.. Determination of honeycomb material properties: existing theories and an alternative dynamic approach. Journal of aerospace engineering 2006;19(3):177-183.

[6] Karakoç, A., Freund, J.. Experimental studies on mechanical properties of cellular structures using nomex® honeycomb cores. Composite Structures 2012;94(6):2017-2024. 
[7] Matter, M., Gmür, T., Cugnoni, J., Schorderet, A.. Identification of the elastic and damping properties in sandwich structures with a low core-to-skin stiffness ratio. Composite Structures 2011;93(2):331-341.

[8] Ip, K.H., Tse, P.C., Lai, T.C.. Material characterization for orthotropic shells using modal analysis and rayleigh-ritz models. Composites Part B: Engineering 1998;29(4):397-409.

[9] Schwaar, M., Gmür, T., Frieden, J.. Modal numerical-experimental identification method for characterising the elastic and damping properties in sandwich structures with a relatively stiff core. Composite Structures 2012;94(7):2227-2236.

[10] Jiang, D., Zhang, D., Fei, Q., Wu, S.. An approach on identification of equivalent properties of honeycomb core using experimental modal data. Finite Elements in Analysis and Design 2014;90:84-92.

[11] El-Sabbagh, A., Steuernagel, L., Ziegmann, G.. Characterisation of flax polypropylene composites using ultrasonic longitudinal sound wave technique. Composites Part B: Engineering 2013;45(1):1164-1172.

[12] Liu, Z., Zhang, X., Mao, Y., Zhu, Y., Yang, Z., Chan, C., et al. Locally resonant sonic materials. Science 2000;289(5485):1734-1736.

[13] Castaings, M., Hosten, B.. Guided waves propagating in sandwich structures made of anisotropic, viscoelastic, composite materials. The Journal of the Acoustical Society of America 2003;113(5):2622-2634.

[14] Scarpa, F., Ouisse, M., Collet, M., Saito, K.. Kirigami auxetic pyramidal core: mechanical properties and wave propagation analysis in damped lattice. Journal of Vibration and Acoustics 2013;135(4):041001.

[15] Droz, C., Zergoune, Z., Boukadia, R., Bareille, O., Ichchou, M.. Vibro-acoustic optimisation of sandwich panels using the wave/finite element method. Composite Structures 2016;

[16] Ranjbar, M., Boldrin, L., Scarpa, F., Neild, S., Patsias, S.. Vibroacoustic optimization of anti-tetrachiral and auxetic hexagonal sandwich panels with gradient geometry. Smart Materials and Structures 2016;25(5):054012.

[17] Antoniadis, I., Chronopoulos, D., Spitas, V., Koulocheris, D.. Hyper-damping properties of a stiff and stable linear oscillator with a negative stiffness element. Journal of Sound and Vibration 2015;346:37-52.

[18] Rose, J.L.. Ultrasonic guided waves in solid media. Cambridge University Press; 2014.

[19] Potel, C., Chotard, T., de Belleval, J.F., Benzeggagh, M.. Characterization of composite materials by ultrasonic methods: modelization and application to impact damage. Composites Part B: Engineering 1998;29(2):159-169.

[20] McDaniel, J.G., Shepard Jr, W.S.. Estimation of structural wave numbers from spatially sparse response measurements. The Journal of the Acoustical Society of America 2000;108(4):1674-1682.

[21] Ichchou, M., Bareille, O., Berthaut, J.. Identification of effective sandwich structural properties via an inverse wave approach. Engineering Structures 2008;30(10):2591-2604.

[22] Papadakis, E.P.. The measurement of ultrasonic velocity. Physical acoustics 1990;19:81-105.

[23] Song, F., Huang, G., Hudson, K.. Guided wave propagation in honeycomb sandwich structures using a piezoelectric actuator/sensor system. Smart Materials and Structures 2009;18(12):125007.

[24] Su, Z., Ye, L., Lu, Y.. Guided lamb waves for identification of damage in composite structures: A review. Journal of sound and vibration 2006;295(3):753-780.

[25] Loosvelt, M., Lasaygues, P.. A wavelet-based processing method for simultaneously determining ultrasonic velocity and material thickness. Ultrasonics 2011;51(3):325-339.

[26] Chimenti, D.. Review of air-coupled ultrasonic materials characterization. Ultrasonics 2014;54(7):1804-1816.

[27] Sikdar, S., Banerjee, S.. Guided wave propagation in a honeycomb composite sandwich structure in presence of a high density core. Ultrasonics 2016;71:86-97.

[28] Chillara, V.K., Lissenden, C.J.. Guided wave mode conversions across waveguide transitions: A study using frequency domain finite element method. In: AIP Conference Proceedings; vol. 1581. 2014, p. 308-315.

[29] Droz, C., Lainé, J.P., Ichchou, M., Inquiété, G.. A reduced formulation for the free-wave propagation analysis in composite structures. Compos Struct 2014;113:134-144.

[30] Nilsson, E., Nilsson, A.. Prediction and measurement of some dynamic properties of sandwich structures with honeycomb and foam cores. Journal of sound and vibration 2002;251(3):409-430.

[31] Mead, D., Markus, S.. The forced vibration of a three-layer, damped sandwich beam with arbitrary boundary conditions. Journal of sound and vibration 1969;10(2):163-175.

[32] Ghinet, S., Atalla, N.. Vibro-acoustic behaviors of flat sandwich composite panels. Transactions of the Canadian Society for Mechanical Engineering 2006;30(4):473-493.

[33] Guillaumie, L.. Vibroacoustic flexural properties of symmetric honeycomb sandwich panels with composite 
faces. Journal of Sound and Vibration 2015;343:71-103.

[34] Baho, O., Zergoune, Z., Ichchou, M., Harras, B., Benamar, R., Troclet, B., et al. On global bendingshear core transition effects for the vibroacoustic of sandwich structures: Analytical and numerical investigations. Composite Structures 2016;154:453-463.

[35] Schriemer, H., Cowan, M., Page, J., Sheng, P., Liu, Z., Weitz, D.. Energy velocity of diffusing waves in strongly scattering media. Physical Review Letters 1997;79(17):3166.

[36] Ciampa, F., Meo, M.. A new algorithm for acoustic emission localization and flexural group velocity determination in anisotropic structures. Composites Part A: Applied Science and Manufacturing 2010;41(12):1777-1786.

[37] Li, F., He, Q., Huang, C., Liu, K., Shao, J., Luo, J.. High frame rate and high line density ultrasound imaging for local pulse wave velocity estimation using motion matching: A feasibility study on vessel phantoms. Ultrasonics 2016;67:41-54. 